\title{
Source Parameter Estimation Method for Assessment of Structural Resiliencies
}

\author{
Zijun Wang and Boming Zhao \\ School of Civil Engineering, Beijing Jiaotong University, Beijing, China \\ Correspondence should be addressed to Boming Zhao; bmzhao@bjtu.edu.cn
}

Received 21 July 2017; Accepted 1 October 2017; Published 30 October 2017

Academic Editor: Xing Ma

Copyright (C) 2017 Zijun Wang and Boming Zhao. This is an open access article distributed under the Creative Commons Attribution License, which permits unrestricted use, distribution, and reproduction in any medium, provided the original work is properly cited.

\begin{abstract}
Assessing structural integrity and sustainability during natural hazards, for instance, strong earthquakes, is an effective way to reduce or even avoid large losses of life and property damage. With the established vulnerability relationships between source parameter and building damage, the seismic resilience of structures can be obtained after the source parameter is estimated in the early stage of an earthquake. For this purpose, we propose a method that employs the $\mathrm{P}$ wave displacement parameter to estimate earthquake magnitude in real time to quick assess the structural resiliencies. By selecting period and amplitude parameter as comparisons, the magnitude estimation formulas are derived, respectively, where the proposed $\mathrm{P}$ wave displacement parameter method is of the highest precision. Through the evolutionary estimation of the $\mathrm{P}$ wave displacement parameter as a function of the time window used, we show that the existing regression relationships can be extended to the large earthquake. Therefore, this paper provides a quick earthquake magnitude estimation method for the establishment of a more reasonable and accurate resilience assessment system for structures.
\end{abstract}

\section{Introduction}

Assessing the structural integrity and sustainability during natural hazards, for instance, strong earthquakes, is an effective way to reduce or even avoid large losses of life and property damage. With the established vulnerability relationships between source parameter and building damage, if we can estimate the earthquake's magnitude before the highintensity shaking occurs, the seismic resilience of structures can be obtained. Earthquake early warning systems [1-4] can provide alerts of impending ground motions within a few seconds to tens of seconds after an earthquake occurs so that appropriate measures can be immediately taken to mitigate seismic risks.

Strong earthquakes occur frequently in Mainland China, where more than 500 earthquakes with magnitude above 6.0 have occurred within 28 provinces from 1900 to the present day. Moreover, the region of earthquake occurrence has increased in size, and events occurred with greater intensity and frequency, meaning that the period of active earthquakes has arrived. These earthquakes destroy buildings and cause severe losses of life and property. For example, more than eighty thousand deaths or disappearances occurred in the $2008 M_{s} 8.0$ Wenchuan earthquake, which was a major tragedy for the Chinese people. Therefore, it is of great significance to seek an approach through scientific research to mitigate the potential threats associated with earthquake hazards.

In this paper, the $\mathrm{P}$ wave displacement parameter $\left(\mathrm{MI}_{d}\right)$ method, which uses data from a single station, is then proposed. We additionally select the representative period $\left(\tau_{c}\right)$ and the amplitude $\left(P_{d}\right)$ parameters, and we compare the relationships between each of these 3 parameters and the magnitude of the aftershocks of the $2008 M_{s} 8.0$ Wenchuan earthquake. In addition, magnitude estimation formulas are derived and the accuracy of these formulas is explored by comparing the estimated magnitude with the catalog magnitude. Furthermore, we investigate to what degree the initial parameters indicate the Wenchuan mainshock magnitude and analyze the relationships among the expanding time window, the proposed $\mathrm{MI}_{d}$ parameter, and the estimated magnitude. With the established vulnerability relationships 
between magnitude and building damage, by quickly predicting the early structural antiseismic capacity from the estimated magnitude, this paper can provide a scientific basis for the establishment of a more reasonable and accurate resilience assessment system for structures.

\section{Dataset}

A robust input database is essential in identifying reliable regression functions for magnitude estimation using a statistical approach. According to the China Strong Motion Net Centre (CSMNC), after the $M_{s} 8.0$ Wenchuan earthquake on May 12, 2008, a total of 383 aftershocks were recorded by the end of 30 September 2008. These events were generated over a rupture length of approximately $300 \mathrm{~km}$ with focal depths ranging from 2 to $20 \mathrm{~km}$, and the records were obtained by strong motion seismographs with a dynamic range of $\pm 2 \mathrm{~g}$, which were mainly installed at free-field sites. The sampling rate was 200 sps.

In this study, we select the mainshock and 43 aftershocks of the $2008 M_{s} 8.0$ Wenchuan earthquake. As criteria, we specify that the selected earthquakes should have magnitude greater than $M_{s}=4.0$ and hypocentral distances less than $150 \mathrm{~km}$. Furthermore, each selected event is required to have at least three records to ensure good station coverage and avoid the path effects. Thus, we use a total of 306 acceleration waveforms from the selected events, which demonstrate a large range of focal depths and mechanisms varying from thrust to strike slip.

Since correct picking of $\mathrm{P}$ arrivals and ensuring the exclusion of $S$ waves from the analysis are prerequisites for accurately calculating the characteristic parameters, we use the three-step $\mathrm{P}$ phase detection method proposed by Wang and Zhao [5], and we double-check the arrival time via manual inspection for each waveform. After performing baseline error correction for the acceleration records, the signals are integrated to velocity records. Moreover, the velocity records are integrated to displacement records, which are required to calculate the parameter values. Then, for realtime applications, a high-pass recursive Butterworth filter with a cutoff frequency of $0.075 \mathrm{~Hz}$ is applied to the vertical components to remove the long-period drift that occurs after integration [6].

\section{Source Parameter Estimation}

3.1. Magnitude Estimation Method. The proposed $\mathrm{P}$ wave displacement parameter $\left(\mathrm{MI}_{d}\right)$ is defined as the integral of the squared high-pass filtered displacement of the vertical component ground motion, which ranges from 0 to 3 seconds of the initial $\mathrm{P}$ wave. The definition of $\mathrm{MI}_{d}$ shows that it is an integral of the squared displacement within the selected time window, which can reflect information with different periods carried by an advancing rupture on a fault plane. As a magnitude estimator, $\mathrm{MI}_{d}$ is a physically fundamental and source-dependent property. Nielsen [7] concluded that the flow rate of elastic energy controls earthquake fracture development and propagation. Fractures with higher initial energy are more likely to continue propagating over long distances and grow into earthquakes with large magnitude.

To verify the validity of the proposed method, we select the two characteristic parameters that have so far proved to be the most robust, namely, $\tau_{c}$ and $P_{d}[8,9]$, for comparison. To correct the calculated $\mathrm{MI}_{d}$ and $P_{d}$ values for the effects of distance, we normalize them to a reference distance of $80 \mathrm{~km}$, which is the average of the hypocentral distances from the analyzed data set, as the way followed by Zollo et al. [10] and Festa et al. [11]. The final values of $\mathrm{MI}_{d}$ and $P_{d}$ are referred to as $\mathrm{MI}_{d}{ }^{\text {Ref }}$ and $P_{d}{ }^{\text {Ref }}$, and they can be obtained after preliminary locations are available. Such locations can be determined using real-time procedures and data from a single station; for example, see the methods proposed by Odaka et al. [12] and Horiuchi et al. [13]. We average the characteristic parameters $\mathrm{MI}_{d}{ }^{\text {Ref }}, \tau_{c}$, and $P_{d}^{\text {Ref }}$ from the multiple observation records associated with each event and assume a linear regression model between the catalog magnitude $M_{s}$ and the averaged parameters as $\log$ (parameter) $=A+B \times M_{s}$, where $A$ and $B$ are constants that are to be determined from the regression analysis.

3.2. Relationships between Magnitude and $M I_{d}, \tau_{c}$, and $P_{d}$. Using the current analytical form, the resulting best-fitting regression relationship between $\mathrm{MI}_{d}{ }^{\mathrm{Ref}}$ and the magnitude is given by

$$
\log \left(\mathrm{MI}_{d}{ }^{\text {Ref }}\right)=-11.175+1.218 \times M_{s} \pm 0.399 .
$$

The individual data points and the average values of $\log \left(\mathrm{MI}_{d}{ }^{\text {Ref }}\right)$ as a function of magnitude $M_{s}$ are shown in Figure 1(a), where SDV means the standard deviation and $R$ stands for the correlation coefficient (similarly hereinafter). The logarithm of the $\mathrm{P}$ wave displacement parameter shows a striking linear correlation with earthquake magnitude within the magnitude range considered $\left(4 \leq M_{s} \leq 6.5\right)$.

The average period of ground motion $\tau_{c}$ is a measurement of the average period of the $\mathrm{P}$ wave within the first few seconds, which is frequently used to estimate magnitude [1]. According to the dataset, the regression relationship between $\tau_{c}$ and magnitude $M_{s}$ is described by

$$
\log \left(\tau_{c}\right)=-1.362+0.246 \times M_{s} \pm 0.148 .
$$

Since $\tau_{c}$ approximately represents the $\mathrm{P}$ wave pulse width, there is a good relationship between the average period of ground motion and the final earthquake magnitude in Figure 1(b).

As for the peak displacement amplitude $P_{d}$ method, the linear regression relationship between the logarithm of $P_{d}{ }^{\text {Ref }}$ and earthquake magnitude $M_{s}$ is established as follows:

$$
\log \left(P_{d}^{\text {Ref }}\right)=-5.076+0.548 \times M_{s} \pm 0.275 .
$$

The regression curve in Figure 1(c) shows that $P_{d}^{\text {Ref }}$ is well correlated with earthquake magnitude. 


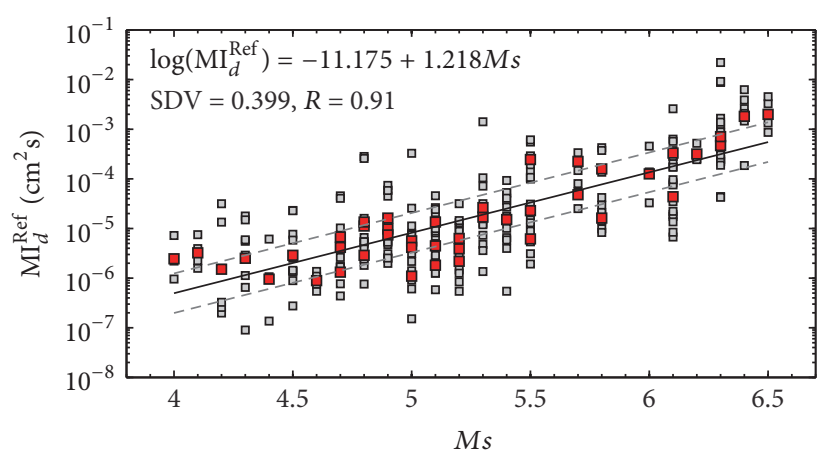

(a)

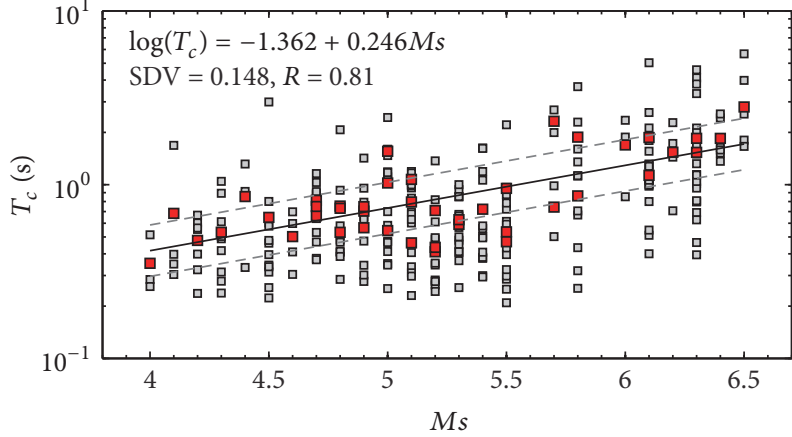

(b)

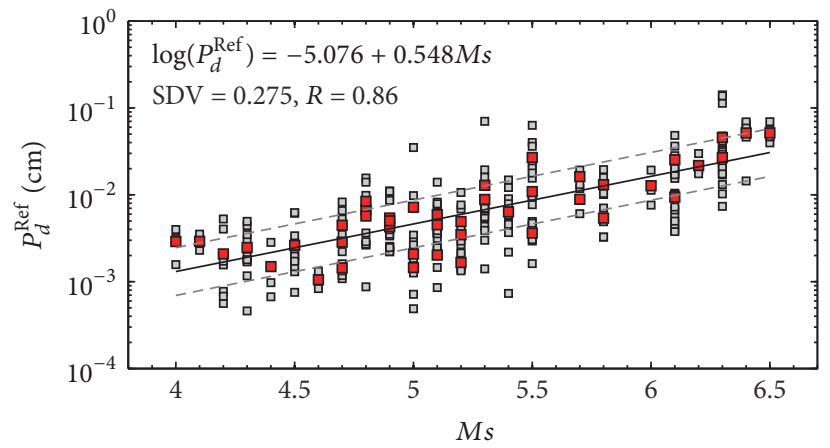

(c)

Figure 1: (a)-(c) show $\mathrm{MI}_{d}^{\text {Ref }}, \tau_{c}$, and $P_{d}^{\text {Ref }}$ calculated from the first 3 seconds of $\mathrm{P}$ wave data (grey squares) and the averaged values (red squares). The magnitude regression relationships determined by this study are shown as solid lines, and the standard deviations are shown as dashed lines.

3.3. Estimated Magnitude Comparison. We can invert the regression results in (1)-(3) to obtain equations for estimating earthquake magnitude from the above 3 characteristic parameters as follows:

$$
\begin{aligned}
M_{\mathrm{MI}_{d}{ }^{\text {Ref }}} & =9.175+0.821 \times \log \left(\mathrm{MI}_{d}^{\mathrm{Ref}}\right) \pm 0.328 \\
M_{\tau_{c}} & =5.537+4.065 \times \log \left(\tau_{c}\right) \pm 0.603 \\
M_{P_{d}{ }^{\text {Ref }}} & =9.262+1.824 \times \log \left(P_{d}^{\text {Ref }}\right) \pm 0.502 .
\end{aligned}
$$

We call these 3 estimated magnitude values $M_{e}$ the $\mathrm{P}$ wave displacement parameter magnitude $M_{\mathrm{MI}_{d}}$ Ref , the average period of ground motion magnitude $M_{\tau_{c}}$, and the peak displacement amplitude magnitude $M_{P_{d} \text { Ref }}$. Figure 2 shows that the $M_{e}$ values calculated from (4) display approximately 1:1 relationships with the catalog magnitude $M_{s}$ using the selected data.

On the $45^{\circ}$ line, $M_{e}=M_{s}$, and the dashed lines illustrate the one-standard-deviation envelopes. Most of the estimated magnitude values fall within one standard deviation of the regression line. The proposed $\mathrm{MI}_{d}{ }^{\mathrm{Ref}}$ is associated with the smallest deviation, that is, 0.328 , whereas the corresponding values for $M_{\tau_{c}}$ and $M_{P_{d}}$ Ref are 0.603 and 0.502 , respectively.

Based on the prediction equations (4), we calculate the differences $\Delta$ between the estimated and catalog magnitude values for each record (i.e., $\Delta=M_{e}-M_{s}$ ), which obey normal distributions. Figure 3 shows histograms that reflect the corresponding frequency distributions. According to the computed probability density curves, the standard deviation associated with the proposed parameter $\mathrm{MI}_{d}{ }^{\text {Ref }}$ is the lowest within the effective estimated range, demonstrating that the $\mathrm{MI}_{d}$ method displays the highest reliability and has apparent advantages over the $\tau_{c}$ and $P_{d}$ methods.

\section{Magnitude Estimation for Large Earthquakes}

The results above imply that the parameters determined from the first 3-second $\mathrm{P}$ wave agree well with the catalog magnitude in the considered range $\left(4 \leq M_{s} \leq 6.5\right)$; thus, the regression equations are practicable to estimate the magnitude for earthquakes under 6.5 reliably. However, for large earthquakes, the use of a limited portion of signals allows capturing only the slip contributions from a limited fault portion, which may result in the underestimation of final earthquake magnitude (i.e., the saturation problem) $[11,14]$.

For the $2008 M_{s} 8.0$ Wenchuan mainshock, there were 32 records available within the criteria (hypocentral distance less than $150 \mathrm{~km}$ ), which provided us with a unique opportunity to investigate the methodologies up to great earthquakes. We first check the extent to which these characteristic parameters could link the mainshock magnitude using the initial 3second $\mathrm{P}$ wave. Then, we analyze the relationships among 


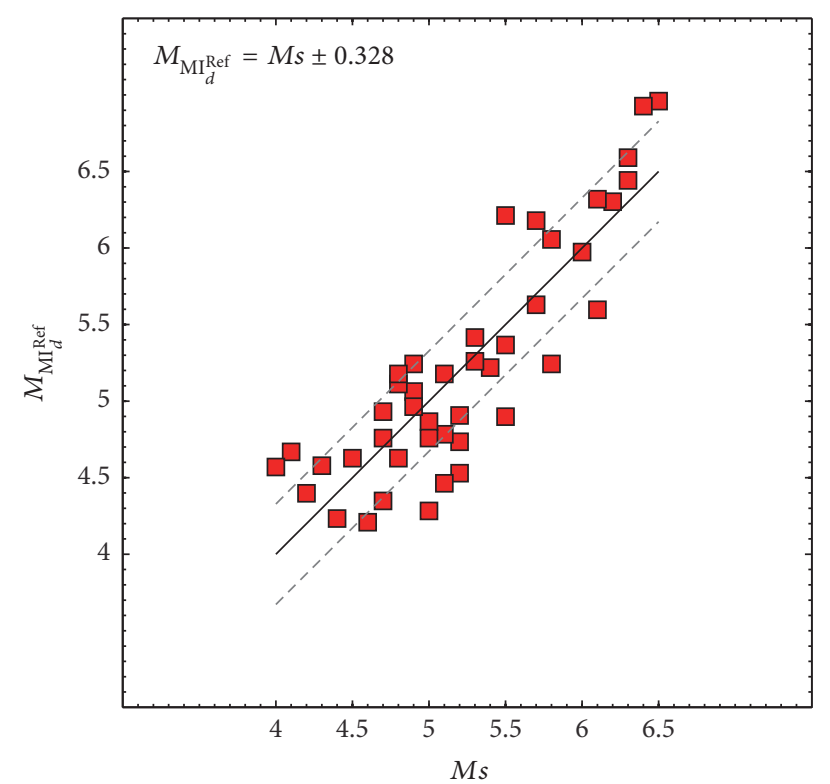

(a)

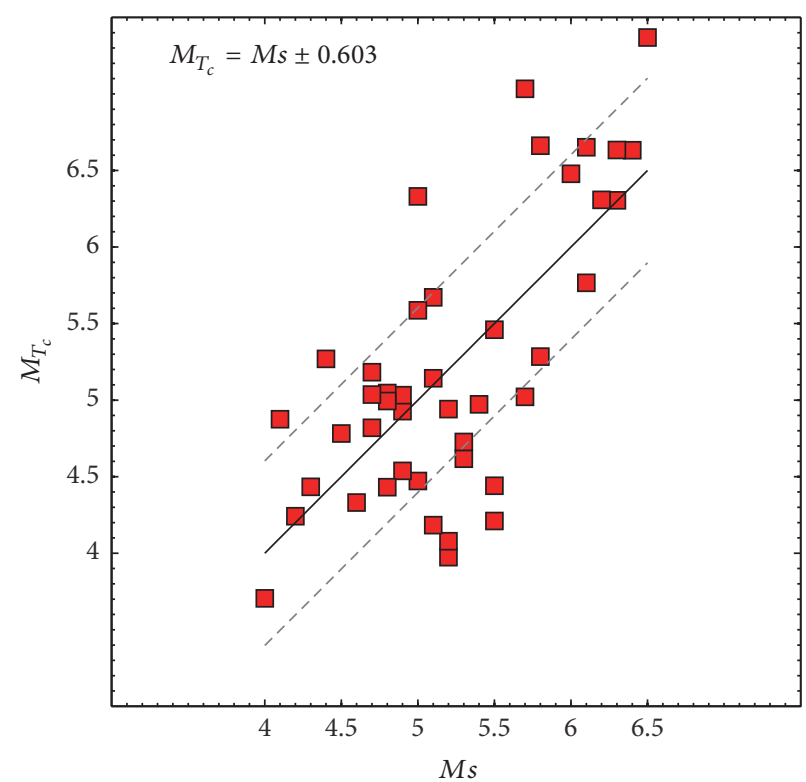

(b)

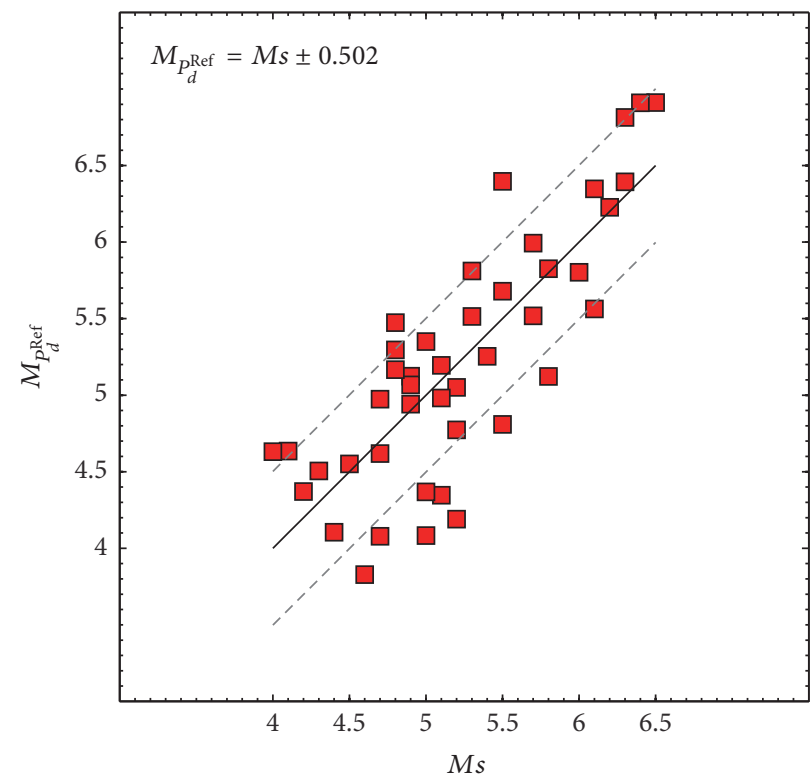

(c)

Figure 2: Magnitude determined using the 3 characteristic parameters versus the catalog magnitude $M_{s}$. Solid lines show the least squares fits, whereas the two dashed lines show the range of one standard deviation.

the increased time window, proposed $\mathrm{MI}_{d}$, and predicted magnitude.

Together with the mainshock and aftershock records, we establish the correlations between $\mathrm{MI}_{d}{ }^{\text {Ref }}, \tau_{c}, P_{d}^{\text {Ref }}$, and magnitude, respectively (see Figure 4), using the procedure discussed above. According to the regressions, the characteristic parameters derived for the mainshock using the 3-second $\mathrm{P}$ wave were all located without the one standard deviation and the anticipated magnitude values were calculated as $M_{\mathrm{MI}_{d}{ }^{\text {Ref }}}=$ $7.18, M_{\tau_{c}}=7.05$, and $M_{P_{d} \text { Ref }}=6.91$, respectively. Although these three parameters all exhibited a saturation problem, the proposed parameter $\mathrm{MI}_{d}$ was relatively more accurate and of the lowest standard deviation, meaning that it could be a reliable and robust magnitude estimator in the earthquake's early stage.

For the saturation problem in predicting large earthquakes, theoretically, a trade-off strategy between time and accuracy can be considered where better estimations could be obtained by enlarging the observation time window to update the characteristic parameters [15-17]. Since our proposed $\mathrm{MI}_{d}$ had the most accurate estimation for the mainshock with 3-second $\mathrm{P}$ wave compared with $\tau_{c}$ and $P_{d}$, we further 


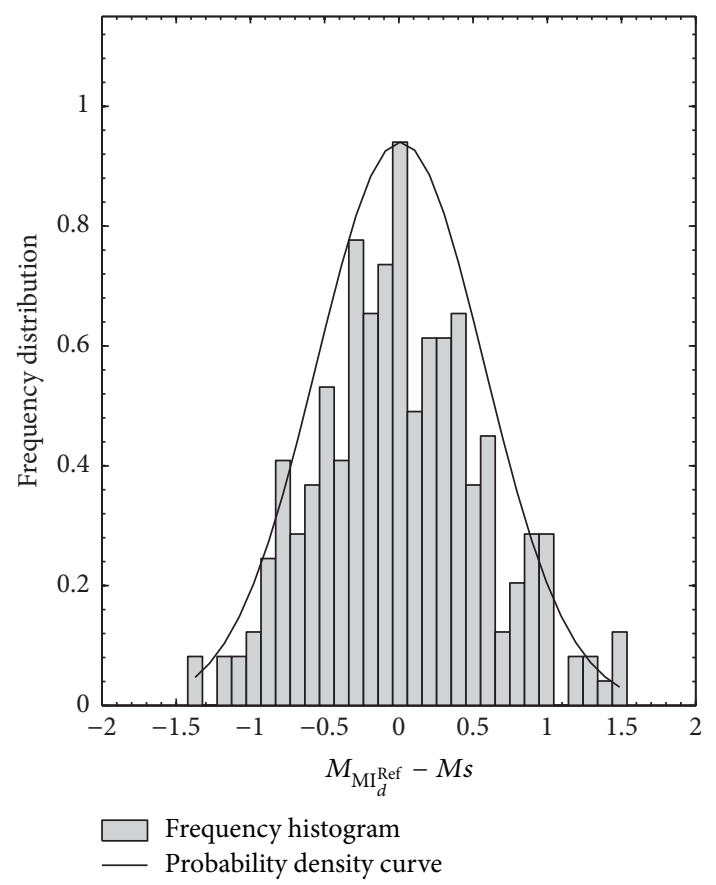

(a)

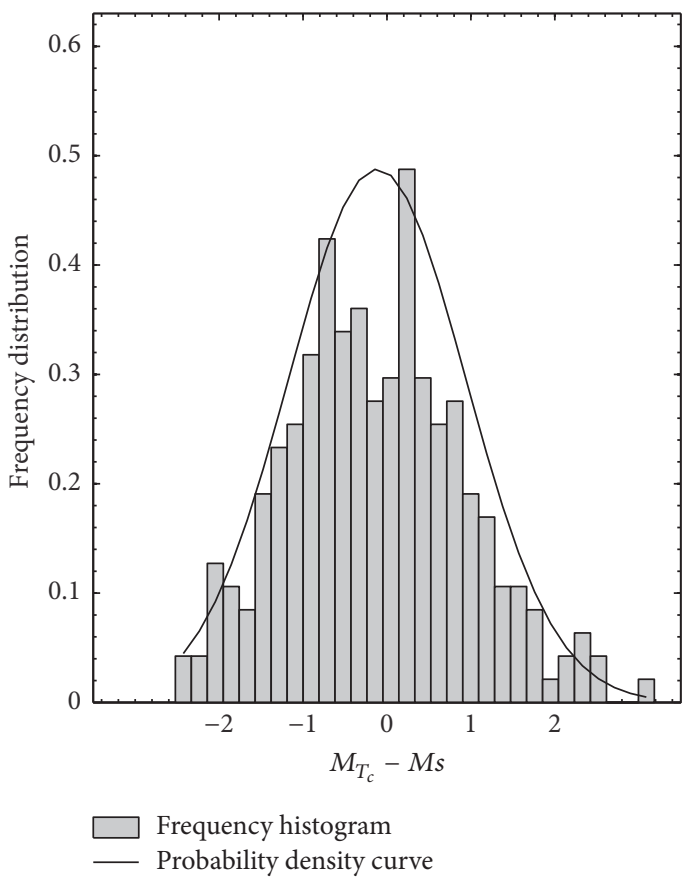

(b)

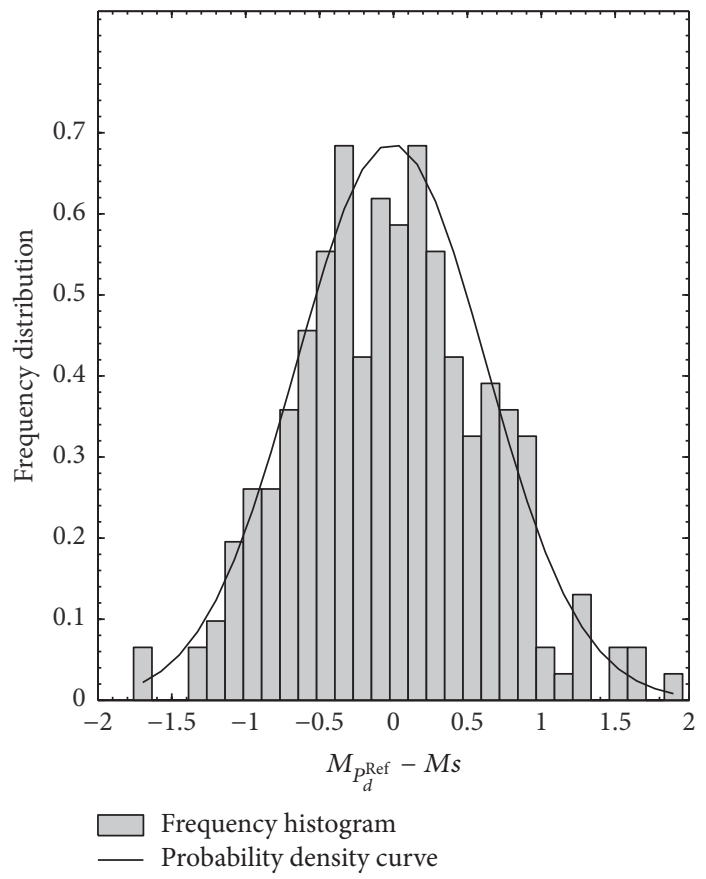

(c)

FIGURE 3: Frequency distribution histograms and the corresponding probability density curves of the estimated magnitude differences using each of the 3 characteristic parameters.

investigate $\mathrm{MI}_{d}$ using different time windows ranging from 3 to 10 seconds for all of the available records.

To avoid the $\mathrm{S}$ wave contamination while increasing the time window, we compute the theoretical $S$ wave arrival times and exclude from our analysis all the stations for which the estimated $S$ wave arrival occurred within the considered time window. Figure 5 shows that the $\mathrm{MI}_{d}^{\text {Ref }}$ magnitude for the Wenchuan mainshock increases with the length of the $\mathrm{P}$ wave records used in determining $\mathrm{MI}_{d}{ }^{\text {Ref }}$. The $\mathrm{MI}_{d}{ }^{\text {Ref }}$ growth pattern implies that $\mathrm{MI}_{d}^{\text {Ref }}$ determined from the first 6-7 seconds of $\mathrm{P}$ wave data may be sufficient to estimate the magnitude of earthquakes of magnitude 8.0. As the window length continues to grow, the magnitude converges at around 8.2. Since the magnitude is estimated only by the 


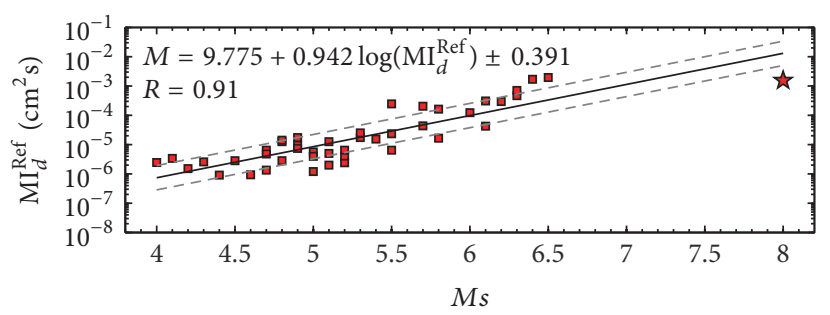

(a)

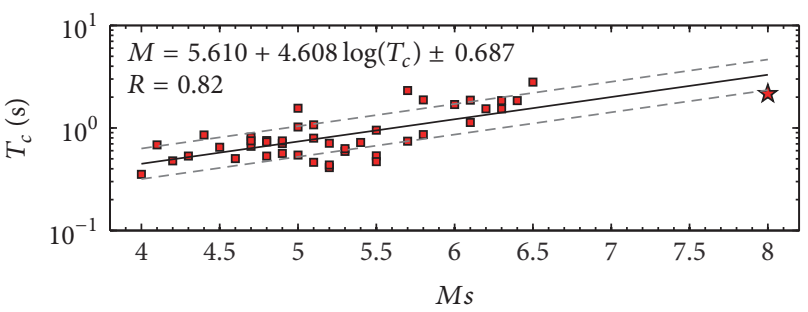

(b)

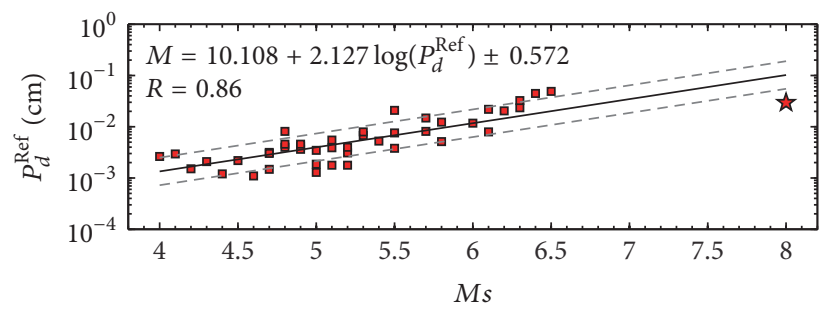

(c)

FIGURE 4: Reestablished regressions of catalog magnitude $M_{s}$ with the 3 characteristic parameters (based on 3 seconds of P wave data) and considering the Wenchuan mainshock (red stars) concurrently. Solid lines show the least squares fits, whereas the two dashed lines show the one-standard-deviation envelopes.

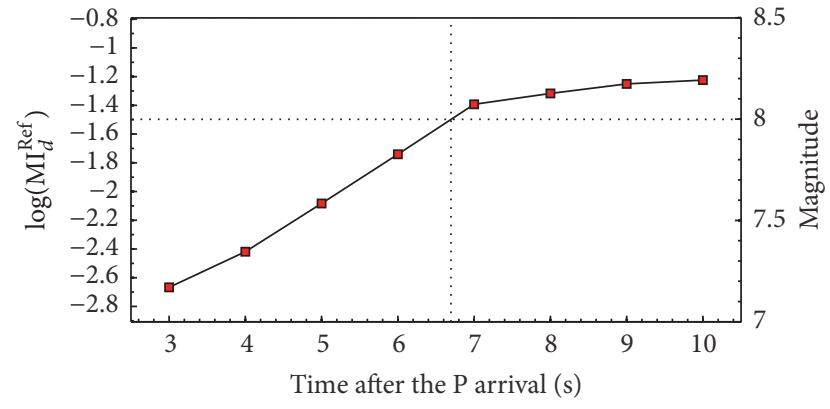

FIGURE 5: Growth in the $\mathrm{MI}_{d}{ }^{\text {Ref }}$ parameter (averaged values) for the Wenchuan mainshock versus the lengths of the initial $P$ wave records used. The corresponding magnitude scale is also represented; this scale is derived using the equation shown in Figure 4(a).

initial $\mathrm{P}$ wave, the results above illustrate that the parameter $\mathrm{MI}_{d}$ may be an accurate and rapid magnitude estimator for earthquakes with magnitude under 6.5. Moreover, it can also provide stable and accurate estimates for large earthquakes if an appropriately increased time window is used.

\section{Discussion and Conclusion}

We proposed a method in terms of the $\mathrm{P}$ wave displacement parameter $\left(\mathrm{MI}_{d}\right)$ to anticipate the magnitude in real time in order to quick assess the seismic resilience of structures. The $\mathrm{MI}_{d}, \tau_{c}$, and $P_{d}$ values for the 43 aftershocks of the 2008 Wenchuan earthquake were calculated and the magnitude estimation formulas from these 3 parameters were derived, respectively. Our result showed that the magnitude obtained from $\mathrm{MI}_{d}$ of 3-second initial $\mathrm{P}$ wave agreed well with the catalog magnitude, where the smallest uncertainty was acquired compared with those of $\tau_{c}$ or $P_{d}$. With the established vulnerability relationships between magnitude and building damage, by quickly predicting the early structural antiseismic capacity from the estimated magnitude, this paper can provide a scientific basis for the establishment of a more reasonable and accurate resilience assessment system for structures.

Using only a small portion of the initial $\mathrm{P}$ wave signals may cause saturation problems in predicting large earthquakes [18]. In the case of the Wenchuan $M_{s} 8.0$ mainshock, using the first 3-second $\mathrm{P}$ wave may underestimate the magnitude by about 0.8 to 1.0 unit with the investigated parameters $\left(M_{\mathrm{MI}_{d}{ }^{\text {Ref }}}=7.18, M_{\tau_{c}}=7.05\right.$, and $\left.M_{P_{d}{ }^{\text {Ref }}}=6.91\right)$; however, the proposed $\mathrm{MI}_{d}$ was of the best performance. To mitigate this problem, several methodologies use a longer time window of $\mathrm{P}$ wave to update magnitude estimates [17, 19]. The evolutionary estimation of $\mathrm{MI}_{d}$ as a function of the time window shows that the existing methodologies and regression relationships can be extended to large earthquakes, and the saturation effect can be removed through the use of time windows of approximately 6-7 seconds with the investigated $\mathrm{MI}_{d}$ parameter.

\section{Conflicts of Interest}

The authors declare that there are no conflicts of interest regarding the publication of this paper.

\section{Acknowledgments}

This research has been supported by the State Key Program of the National Natural Science Foundation of China (Grant no. U1434210), the National Natural Science Foundation of China (Grants nos. 51778046 and 51278045), and the Project of Shenzhen Metro Group Co., Ltd. (no. SZ-CGMKY001/2014). 


\section{References}

[1] H. Kanamori, "Real-time seismology and earthquake damage mitigation," Annual Review of Earth and Planetary Sciences, vol. 33, no. 1, pp. 195-214, 2005.

[2] G. Wurman, R. M. Allen, and P. Lombard, "Toward earthquake early warning in northern California," Journal of Geophysical Research: Atmospheres, vol. 112, no. B8, 2007.

[3] R. M. Allen, P. Gasparini, O. Kamigaichi, and M. Böse, “The status of earthquake early warning around the World: an introductory overview," Seismological Research Letters, vol. 80, no. 5, pp. 682-693, 2009.

[4] H. S. Kuyuk, R. M. Allen, H. Brown, M. Hellweg, I. Henson, and D. Neuhauser, "Designing a network-based earthquake early warning algorithm for California: ElarmS-2," Bulletin of the Seismological Society of America, vol. 104, no. 1, pp. 162-173, 2014.

[5] Z. Wang and B. Zhao, "Automatic event detection and picking of P, S seismic phases for earthquake early warning and application for the 2008 Wenchuan earthquake," Soil Dynamics and Earthquake Engineering, vol. 97, pp. 172-181, 2017.

[6] Y.-M. Wu and L. Zhao, "Magnitude estimation using the first three seconds P-wave amplitude in earthquake early warning," Geophysical Research Letters, vol. 33, no. 16, Article ID L16312, 2006.

[7] S. Nielsen, "Can earthquake size be controlled by the initial seconds of rupture?" Earthquake Early Warning Systems, pp. 920, 2007.

[8] M. Yamada and J. Mori, "Using $\tau \mathrm{C}$ to estimate magnitude for earthquake early warning and effects of near-field terms," Journal of Geophysical Research: Solid Earth, vol. 114, no. 5, p. B05301, 2009.

[9] W. Wang, S. Ni, Y. Chen, and H. Kanamori, "Magnitude estimation for early warning applications using the initial part of P waves: A case study on the 2008 Wenchuan sequence," Geophysical Research Letters, vol. 36, no. 16, 2009.

[10] A. Zollo, M. Lancieri, and S. Nielsen, "Earthquake magnitude estimation from peak amplitudes of very early seismic signals on strong motion records," Geophysical Research Letters, vol. 33, no. 23, Article ID L23312, 2006.

[11] G. Festa, A. Zollo, and M. Lancieri, "Earthquake magnitude estimation from early radiated energy," Geophysical Research Letters, vol. 35, no. 22, Article ID L22307, 2008.

[12] T. Odaka, K. Ashiya, S. Tsukada, S. Sato, K. Ohtake, and D. Nozaka, "A new method of quickly estimating epicentral distance and magnitude from a single seismic record," Bulletin of the Seismological Society of America, vol. 93, no. 1, pp. 526532, 2003.

[13] S. Horiuchi, H. Negishi, K. Abe, A. Kamimura, and Y. Fujinawa, "An automatic processing system for broadcasting earthquake alarms," Bulletin of the Seismological Society of America, vol. 95, no. 2, pp. 708-718, 2005.

[14] C. Cauzzi, Y. Behr, J. Clinton, P. Kästli, L. Elia, and A. Zollo, “An open-source earthquake early warning display," Seismological Research Letters, vol. 87, no. 3, pp. 737-742, 2016.

[15] P. Rydelek, C. Wu, and S. Horiuchi, "Comment on "Earthquake magnitude estimation from peak amplitudes of very early seismic signals on strong motion records" by Aldo Zollo, Maria Lancieri, and Stefan Nielsen," Geophysical Research Letters, vol. 34, no. 20, Article ID L20302, 2007.

[16] I. Iervolino, M. Giorgio, C. Galasso, and G. Manfredi, "Uncertainty in early warning predictions of engineering ground motion parameters: what really matters?" Geophysical Research Letters, vol. 36, no. 5, Article ID L00B06, 2009.

[17] S. Colombelli, A. Zollo, G. Festa, and H. Kanamori, "Early magnitude and potential damage zone estimates for the great Mw 9 Tohoku-Oki earthquake," Geophysical Research Letters, vol. 39, no. 22, Article ID L22306, 2012.

[18] C. Satriano, Y.-M. Wu, A. Zollo, and H. Kanamori, "Earthquake early warning: Concepts, methods and physical grounds," Soil Dynamics and Earthquake Engineering, vol. 31, no. 2, pp. 106118, 2011.

[19] O. Kamigaichi, M. Saito, K. Doi et al., "Earthquake early warning in Japan: Warning the general public and future prospects,' Seismological Research Letters, vol. 80, no. 5, pp. 717726, 2009. 


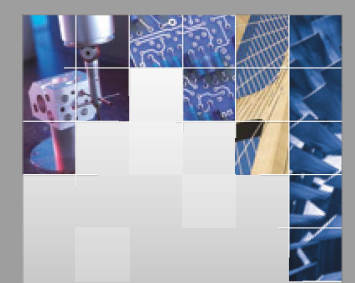

\section{Enfincering}
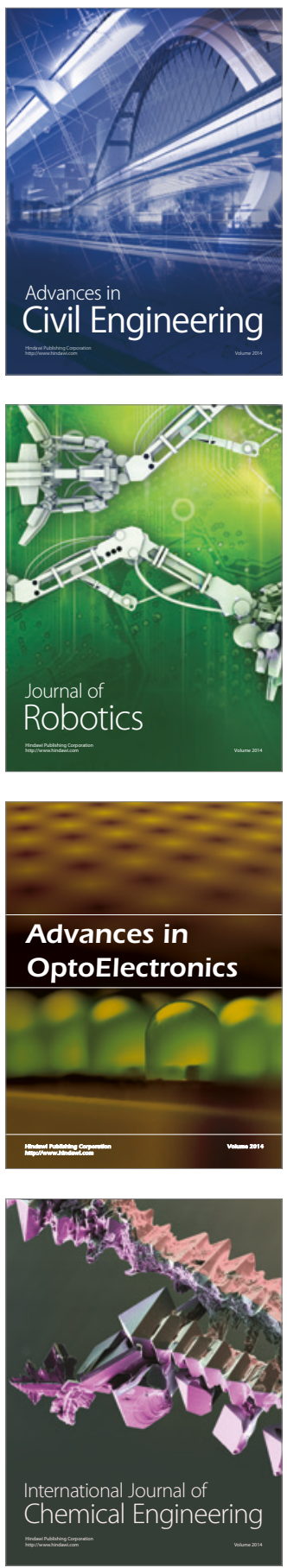

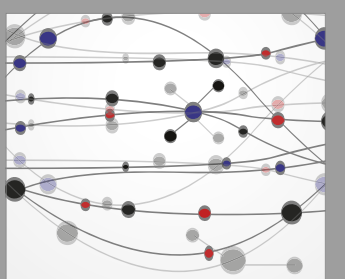

The Scientific World Journal

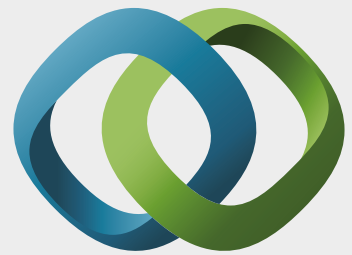

\section{Hindawi}

Submit your manuscripts at

https://www.hindawi.com
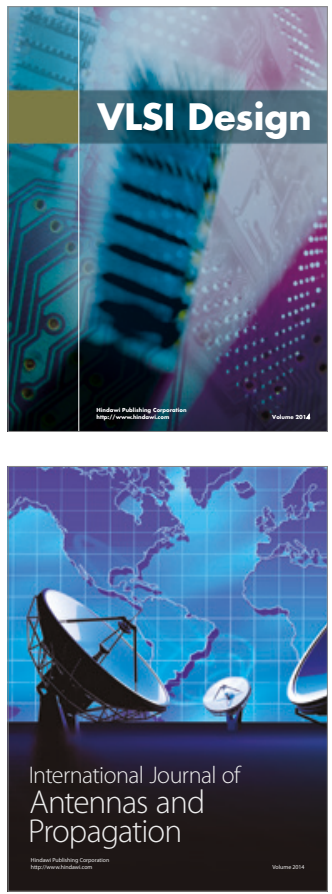

\section{Rotating}

Machinery
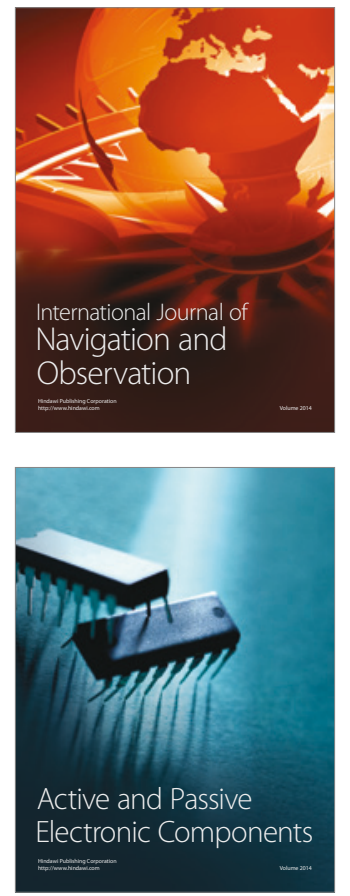
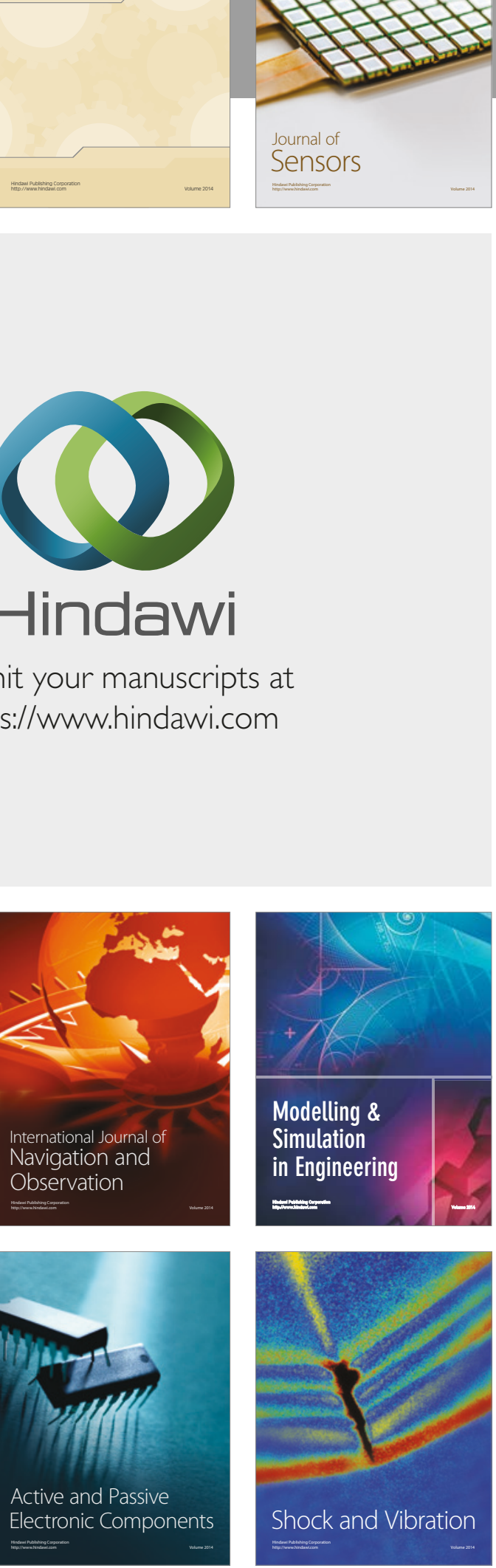
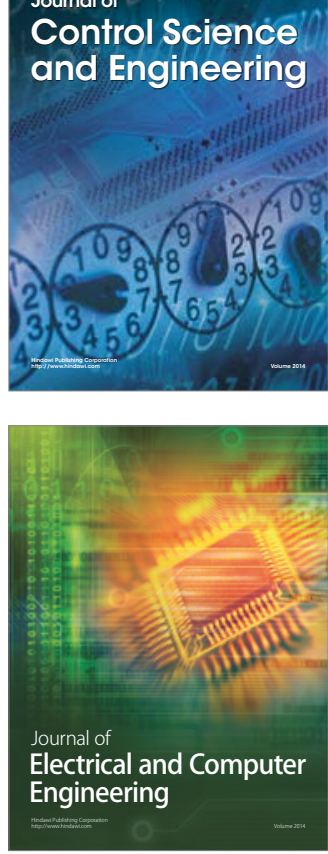

Distributed

Journal of

Control Science

and Engineering
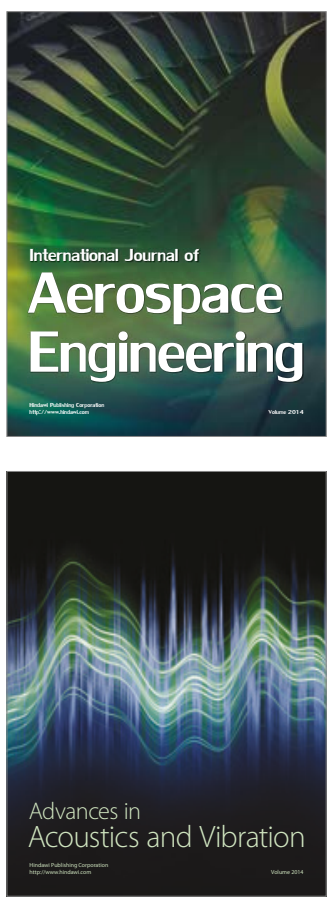

Sensor Networks 